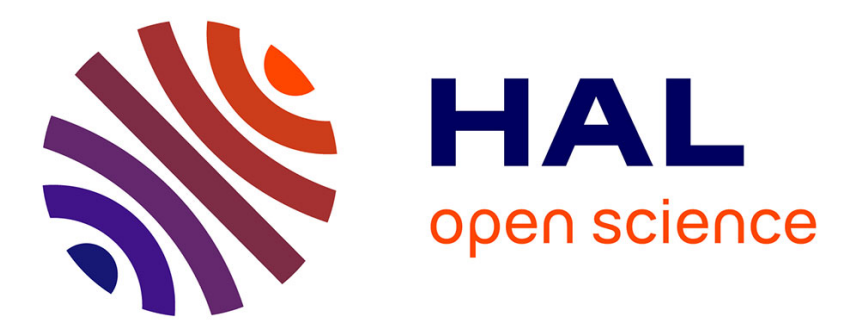

\title{
On optimal growth models when the discount factor is near 1 or equal to 1
}

\author{
Cuong Le Van, Lisa Morhaim
}

\section{To cite this version:}

Cuong Le Van, Lisa Morhaim. On optimal growth models when the discount factor is near 1 or equal to 1. International Journal of Economic Theory, 2006, 2 (1), pp.55-76. 10.1111/j.1365-2966.2006.0024.x . halshs-00096034

\section{HAL Id: halshs-00096034 \\ https://shs.hal.science/halshs-00096034}

Submitted on 27 Sep 2006

HAL is a multi-disciplinary open access archive for the deposit and dissemination of scientific research documents, whether they are published or not. The documents may come from teaching and research institutions in France or abroad, or from public or private research centers.
L'archive ouverte pluridisciplinaire HAL, est destinée au dépôt et à la diffusion de documents scientifiques de niveau recherche, publiés ou non, émanant des établissements d'enseignement et de recherche français ou étrangers, des laboratoires publics ou privés. 


\title{
On Optimal Growth Models when the Discount Factor is near 1 or equal to 1
}

\author{
Cuong Le Van (CNRS-CERMSEM)* \\ Lisa Morhaim (CERMSEM, University of Paris 1$)^{* \dagger}$
}

December 2005

\begin{abstract}
The aim of this paper is to fill the gap between intertemporal growth models when the discount factor is close to one and when it equals one. We show that the value function and the policy function are continuous with respect both to the discount factor $\beta$ and the initial stock of capital $x_{0}$. We prove that the optimal policy $g^{\beta}\left(x_{0}\right)$ is differentiable and that $D g^{\beta}\left(x_{0}\right)$ is continuous with respect to $\left(\beta, x_{0}\right)$. As a by-product, a global turnpike result is proved.
\end{abstract}

Keywords. Optimal growth, discount factor, value function, policy function, differentiability, turnpike.

JEL Classification. C61, O41.

\section{Introduction}

A large part of the litterature on intertemporal models of consumption and capital accumulation has focused on the existence and dynamical properties of optimal solutions to optimization problems. Dynamic programming gives an attractive methodology for studying the behavior of optimal paths as long as the information about optimal solutions is summarized in the policy function $g$. The

*CERMSEM, EP 1737 du CNRS, Université de Paris I, Maison des Sciences Economiques, 106-112 Bd de l'Hôpital 75647 Paris Cedex 13, France. E-mail: levan@univ-paris1.fr, morhaim@univ-paris1.fr. Tel: 331440783 00. Fax: 33144078301.

$\dagger$ The authors are grateful to Manuel Santos, Kevin Reffet and an anonymous referee for many remarks and comments. 
properties of the optimal policy and the value function are well-known in the case when the discount factor $\beta$ is strictly less than one.

Benveniste and Scheinkman[3] have proved the differentiability of the value function. Araujo and Scheinkman[2] under usual conditions and a very strong condition(the so-called "dominant diagonal blocks" conditions) show the equivalence between smoothness with respect to the initial capital stock of the optimal policy and turnpike property of the optimal path. Blot and Crettez[4] have given some alternative sufficient conditions (different from "dominant diagonal blocks" conditions) to have the $C^{k}$ - differentiability vis-à-vis the initial stock of capital, the discount factor $\beta<1$, and a parameter which lies in a Banach space. For one-sector models, Araujo has given some conditions for the monotonically increasing policy function to be differentiable and (what is the same) for the value function to be twice differentiable with respect to the initial capital stock. He has also shown that if the return function $u$ is $C^{3}$, the value function is not necessarily $C^{2}$. Although Santos[13], [15] has shown the differentiability of policy functions, his analysis does not include the case where the discount factor equals one. In particular, the proof in Santos [15] is crucially based on the fact that the discount factor is strictly less than one. The same author (see [14]) proves that the policy function could be $C^{1}$ with respect to the initial capital stock and the discount factor. In a stochastic setting, Santos and Vigo-Agular (see [16]) prove that the optimal policy could be $C^{1}$ with respect to capital stock and the initial shocks. Another proof of Santos's [13], [15] theorem has been given by Montrucchio[12]. Note that Santos[13] uses results from Boldrin and Montrucchio [5]. Scheinkman[17] and Mc Kenzie[9] have shown for $\beta$ close to 1, that the turnpike property holds: the optimal path converges to the stationary state. Note also that Yano[19] is concerned with similar issues.

When $\beta=1$, we know by Gale[7] that the optimal path converges. But we do not know anything about the continuity with respect to $\beta$ of the value and policy functions at $\beta=1$, and about whether the policy function is differentiable or not when $\beta=1$. Here, we show that the value function and policy function are continuous with respect both to the discount factor $\beta$ and the initial stock of capital $x$. Moreover, we show that the optimal policy $g^{\beta}($.$) is differentiable and$ that $D g^{\beta}(x)$ is continuous with respect to $(\beta, x)$, whereas Santos[13], on the case when $\beta<1$ has shown the $C^{0}$-differentiability with respect to $x$, or jointly $C^{1}$ with respect to the capital stock and the discount factor.

Dana and Le Van [6] have introduced the value function for the case $\beta=1$ :

$$
V^{1}\left(x_{0}\right)=\max \left\{\sum_{t=0}^{+\infty}\left(u\left(x_{t}, x_{t+1}\right)-u(\underline{x}, \underline{x})\right) ; x_{t+1} \in D\left(x_{t}\right), \forall t, x_{0} \text { is given }\right\}
$$


where $\underline{x}$ is defined by $u(\underline{x}, \underline{x})=\max _{x \in D(x)} u(x, x)$ and $D$ is the technology correspondence.

They have shown that under usual assumptions the value function is an upper semi-continuous function that satisfies the Bellman equation and that it is the only upper semi-continuous solution to the Bellman equation.

Obviously, it is clear that if the problem $V^{\beta}\left(x_{0}\right)=\max \left\{\sum_{t=0}^{+\infty} \beta^{t} u\left(x_{t}, x_{t+1}\right) ; x_{t+1} \in\right.$ $D\left(x_{t}\right), \forall t, x_{0}$ is given $\}$ is considered, it can not converge to $V^{1}$ when $\beta$ converges to one. But it can be shown that for $\beta$ near one, there exists a stationary state $x^{\beta}$ for this problem. In order to have a consistent formalization, we write the problem in the following way:

$$
V^{\beta}\left(x_{0}\right)=\max \left\{\sum_{t=0}^{+\infty} \beta^{t}\left(u\left(x_{t}, x_{t+1}\right)-u\left(x^{\beta}, x^{\beta}\right)\right) ; x_{t+1} \in D\left(x_{t}\right), \forall t, x_{0} \text { is given }\right\}
$$

for $\beta$ in a neighborhood $\left[\beta_{0}, 1\right]$ of 1 such that the stationary state $x^{\beta}$ exists.

The goal of this paper is to fill the gap between $\beta<1$ and $\beta=1$. We show that:

- $V^{\beta}\left(x_{0}\right)$ is continuous with respect to $\left(\beta, x_{0}\right) \in\left[\beta_{0}, 1\right] \times X$, where $X$ is the compact space of states.

- $g^{\beta}\left(x_{0}\right)$ is continuous with respect to $\left(\beta, x_{0}\right) \in\left[\beta_{0}, 1\right] \times X$.

- Under certain assumptions as $\alpha$-concavity, $g^{\beta}\left(x_{0}\right)$ is differentiable and continuous with respect to $\left(\beta, x_{0}\right) \in\left[\beta_{0}, 1\right] \times X$. To obtain this result, we combine and extend results of Santos[13] and Montrucchio[12]. But the extension requires the continuity in $(\beta, x)$ of the value function when $\beta$ is close or equal to 1 . It also requires the existence of a sequence of functions, continuous in $(\beta, x), C^{2}$ and concave in $x$, which converges uniformly in $(\beta, x)$ to the value-function $V^{\beta}(x)$ (see Lemma 1 ).

- As a by-product, we finally obtain a turnpike result for $\beta$ close to 1 . The idea is, as soon as we now know that $D g^{\beta}\left(x^{\beta}\right)$ converges to $D g^{1}(\underline{x})$ and that the turnpike result holds for $\beta=1$, then it can be deduced that the eigenvalues of $D g^{1}(\underline{x})$ are of modulus strictly less than 1 . Then, there exists a neighborhood of 1 , say $\left[\beta_{0}^{\prime}, 1\right]$ such that $\forall \beta \in\left[\beta_{0}^{\prime}, 1\right]$, the eigenvalues of $D g^{\beta}\left(x^{\beta}\right)$ are also of modulus strictly less than 1 . We then first show, thanks to the continuity with respect to both $\beta$ and $x_{0}$, that there exists an $\varepsilon$ such that if $\left\|x^{\beta}-x_{0}\right\| \leq \varepsilon$, then the optimal path starting from $x_{0},\left\{g^{\beta, t}\left(x_{0}\right)\right\}_{t}$, converges to $x^{\beta}$, for any $\beta$ in $\left[\beta_{0}^{\prime}, 1\right]$. Now, if $x_{0}$ does not satisfy $\left\|x^{\beta}-x_{0}\right\| \leq \varepsilon$, then by Scheinkman's Visit Lemma, there exists 
$\beta(\varepsilon)$ such that for any $\beta \geq \beta(\varepsilon)$, there exists $T$ such that $g^{\beta, T}\left(x_{0}\right)$ satisfies $\left\|x^{\beta}-g^{\beta, T}\left(x_{0}\right)\right\| \leq \varepsilon$. Then the sequence $g^{\beta, t}\left(g^{\beta, T}\left(x_{0}\right)\right)$ converges to $x^{\beta}$. From the Mangasarian Lemma, the whole sequence $\left\{g^{\beta, t}\left(x_{0}\right)\right\}_{t}$ is optimal. Obviously, this one converges to $x^{\beta}$. Summing up, for any $x_{0} \in X$, for any $\beta \in\left[\max \left(\beta_{0}, \beta(\varepsilon)\right), 1\right]$, the turnpike property holds.

One can wonder why Santos[13] and Montrucchio[12] obtain their results only when $\beta<1$. The approach used by Santos [14], [15], and Montrucchio[12] is based on the contraction property which holds only when $\beta<1$. A careful inspection of the proof in Santos[13] reveals that his approach does not require contraction property. What is important is the continuity of the value function and of the optimal policy. The properties hold when $\beta<1$. But when $\beta \leq 1$, some more proofs are necessary. It will be done in Section 4 of our paper.

The paper is organized as follows: Section 2 deals with the model; in Section 3 , we consider the no-discounting case; Section 4 deals with continuity of the value function and the optimal policy with respect both to $\beta$ and $x$; Section 5 gives the crucial results of existence and continuity of $D g^{\beta}(x)$ with both $\beta$ and $x$; In Remark 4 we finally give a very straightforward proof for the turnpike result as a by-product of the differentiability of the policy function.

\section{The Model}

As in Dana and Le Van (1990), we consider a triplet $(X, D, u)$ and make the following assumptions:

(H1) $X$ is a compact convex subset of $\mathbb{R}_{+}^{n}, n \geq 1$, with nonempty interior, that contains 0 .

(H2) $D$ is a continuous set-valued correspondence from $X$ into a compact set of $X$, with nonempty convex compact images. Its graph is convex. $0 \in D(0)$.

(H3) $\forall x_{0} \geq 0, x_{0} \neq 0, \exists y_{0}>>0, y_{0} \in D\left(x_{0}\right)$.

(H4) (free disposal) If $y \in D(x), x^{\prime} \geq x, y^{\prime} \leq y$, then $y^{\prime} \in D\left(x^{\prime}\right)$.

(H5) (existence of an expansible stock) There exists $(x, y), y \in D(x)$ such that $y>>x$.

(H6) The utility function $u: \operatorname{graph} D \rightarrow \mathbb{R}$ is a strictly concave $C^{2}$ function, $u(x, y)$ is increasing in $x$, decreasing in $y^{1}$.

Note that (H4) and (H5) ensure that intgraph ${ }^{2}$ is nonempty. Moreover,

\footnotetext{
${ }^{1}$ As in Santos [14], we say that the utility function is $C^{2}$ in the graph of $D$ if it has a $C^{2}$ extension on an open set.

${ }^{2}$ We denote by intgraph $D$ the interior of graph $D$ with the induced topology.
} 
by (H2) and Kakutani's theorem, the set of fixed-points of $D$ is nonempty.

We define the following program:

$$
\begin{aligned}
\bar{u}= & \operatorname{Max} u(x, x) \\
& \text { s.t. } x \in D(x)
\end{aligned}
$$

Then from (H5):

$$
\begin{array}{cc}
\bar{u}=\operatorname{Max} & u(x, x) \\
\text { s.t. } & y \in D(x) \\
& y \geq x
\end{array}
$$

Throughout this paper, we denote by $\tilde{x}$ a sequence in $X$, i.e., $\tilde{x}=\left(x_{0}, x_{1}, \ldots, x_{t}, \ldots\right)$ with $x_{t}$ in $\mathrm{X}$, for any $t$.

Definition $1 A$ program is a sequence $\tilde{x} \in X^{\mathbb{N}}$ such that $\forall t, x_{t+1} \in D\left(x_{t}\right)$. $\Gamma\left(x_{0}\right)$ is the set of programs with initial stock $x_{0}$.

Observe that $\Gamma$ is a continuous correspondence since $D$ is continuous.

\section{Optimal growth without discounting}

Definition 2 Let $\underline{x}=\operatorname{argmax}\{u(x, x), x \in D(x)\}$. A program $\tilde{x} \in \Gamma\left(x_{0}\right)$ is $\operatorname{good}^{3}$ if

$$
\lim _{T \rightarrow+\infty} \sum_{t=0}^{T}\left(u\left(x_{t}, x_{t+1}\right)-u(\underline{x}, \underline{x})\right) \text { exists in } \mathbb{R} .
$$

Let us denote by $\Gamma_{G}\left(x_{0}\right)$ the set of good programs starting from $x_{0}$.

Proposition 1 If $\tilde{x}$ is a good program, then $\lim _{t} x_{t}=\underline{x}$

$$
\text { Proof: See Gale[7] }
$$

\footnotetext{
${ }^{3}$ This is the definition in Le Van and Dana[8]. It can be checked that it is equivalent to the definition given by Gale[7]: $\tilde{x} \in \Gamma\left(x_{0}\right)$ is good if for any $\tilde{x}^{\prime} \in \Gamma\left(x_{0}\right)$, one has $\liminf _{T \rightarrow+\infty} \sum\left(u\left(x_{t}, x_{t+1}\right)-u\left(x_{t}^{\prime}, x_{t+1}^{\prime}\right)\right)>-\infty$.
} 
Definition 3 A program $\tilde{x}^{*} \in \Gamma\left(x_{0}\right)$ is optimal ${ }^{4}$ if we have:

$$
\forall \tilde{x} \in \Gamma\left(x_{0}\right), \lim _{T \rightarrow+\infty} \sum_{t=0}^{T}\left(u\left(x_{t}^{*}, x_{t+1}^{*}\right)-u(\underline{x}, \underline{x})\right) \geq \lim _{T \rightarrow+\infty} \sum_{t=0}^{T}\left(u\left(x_{t}, x_{t+1}\right)-u(\underline{x}, \underline{x})\right) .
$$

Proposition 2 The stationary program $\underline{\tilde{x}}=(\underline{x}, \underline{x}, \ldots)$ is an optimal program from $\underline{x}$.

Proof: See Gale [7] or Le Van and Dana [8].

Definition 4 Let us define $\gamma(\tilde{x}):=\lim _{T \rightarrow+\infty} \sum_{t=0}^{T}\left[u\left(x_{t}, x_{t+1}\right)-u(\underline{x}, \underline{x})\right]$.

Then the optimal growth problem becomes:

$$
\max _{\tilde{x} \in \Gamma\left(x_{0}\right)} \gamma(\tilde{x})
$$

Proposition 3 If $\Gamma_{G}\left(x_{0}\right) \neq \emptyset$ then there exists an optimal program from $x_{0}$. It is a good program.

Proof: See Dana and Le Van[6].

Proposition 4 Assume $\Gamma_{G}\left(x_{0}\right) \neq \emptyset$. Let $\tilde{x}^{*} \in \Gamma\left(x_{0}\right)$ satisfy $\forall t,\left(x_{t}^{*}, x_{t+1}^{*}\right) \in$ intgraphD. Then $\tilde{x}^{*}$ is optimal if and only if it satisfies the Euler equation:

$$
\forall t, u_{2}\left(x_{t}^{*}, x_{t+1}^{*}\right)+u_{1}\left(x_{t+1}^{*}, x_{t+2}^{*}\right)=0 .
$$

Proof: See Dana and Le Van[6].

\section{About the continuity of the value function and the policy function when $\beta$ is close to 1 or equals 1}

Let us consider the following problem

\footnotetext{
${ }^{4}$ This is the definition of an optimal program in the sense of Le Van and Dana[8]. An optimum in the sense of Gale is optimal in the sense of Le Van and Dana[8] and the two definitions coincide when $\Gamma_{G}\left(x_{0}\right) \neq \emptyset$.
} 


$$
\left\{\begin{array}{cl}
\text { Maximize } \sum_{t=0}^{+\infty} \beta^{t} u\left(x_{t}, x_{t+1}\right) \\
\text { s.t. } \quad \forall t, x_{t+1} \in D\left(x_{t}\right) \\
& x_{0} \text { is given } \\
& \beta \in(0,1) .
\end{array}\right.
$$

Proposition 5 Assume the Hessian of $u$ at $(\underline{x}, \underline{x})$ is negative definite. Then there exists a neighborhood of $1,\left[\beta_{0}, 1\right]$ such that $\forall \beta \in\left[\beta_{0}, 1\right]$, there exists a unique steady state $x^{\beta}$. Moreover $\forall \beta \in\left[\beta_{0}, 1\right], \beta \rightarrow x^{\beta}$ is continuous. In particular, one has $\lim _{\beta \rightarrow 1} x^{\beta}=\underline{x}$.

Proof: Consider the equation:

$$
u_{2}\left(x^{\beta}, x^{\beta}\right)+\beta u_{1}\left(x^{\beta}, x^{\beta}\right)=0
$$

Differentiate it and obtain:

$(*)\left[u_{21}\left(x^{\beta}, x^{\beta}\right)+\beta u_{12}\left(x^{\beta}, x^{\beta}\right)+u_{22}\left(x^{\beta}, x^{\beta}\right)+\beta u_{11}\left(x^{\beta}, x^{\beta}\right)\right] d x^{\beta}+u_{1}\left(x^{\beta}, x^{\beta}\right) d \beta=0$.

Consider $z_{(2 n)}=\left(z_{(n)}, z_{(n)}\right) \neq 0$. For $\beta=1$, one has $x^{\beta}=\underline{x}$ and since the Hessian matrix $H$ is negative definite:

$$
{ }^{t} z_{(2 n)} H z_{(2 n)}={ }^{t} z_{(n)}\left(2 u_{21}(\underline{x}, \underline{x})+u_{22}(\underline{x}, \underline{x})+u_{11}(\underline{x}, \underline{x})\right) z_{(n)}<0
$$

The matrix $\left[u_{21}(\underline{x}, \underline{x})+u_{12}(\underline{x}, \underline{x})+u_{22}(\underline{x}, \underline{x})+u_{11}(\underline{x}, \underline{x})\right]$ is invertible. Since for $\beta=1,\left(\mathcal{E}^{\prime}\right)$ has a unique solution $\underline{x}$, then by the Implicit Function Theorem, there exists a neighborhood of $\beta \in\left[\beta_{0}^{\prime}, 1\right]$, and a neighborhood $\mathcal{V}(\underline{x})$ of $\underline{x}$ such that there is a unique solution $x^{\beta}$, which is continuous with respect to $\beta$. That is $x^{\beta} \rightarrow \underline{x}$ when $\beta$ converges to 1 . We claim that there exists $\beta_{0} \geq \beta_{0}^{\prime}$ such that $\forall \beta \in\left[\beta_{0}, 1\right], x^{\beta}$ is unique. Indeed, suppose the contrary. There will be a sequence $\beta_{n} \rightarrow 1$, with another steady state $x_{1}^{\beta_{n}} \notin \mathcal{V}(\underline{x}), \forall n$. But $x_{1}^{\beta_{n}} \rightarrow \underline{x}$, which is a contradiction since there will be two steady states in $\mathcal{V}(\underline{x})$.

In the remaining of the paper, we will add:

(H7) The Hessian $D^{2} u(\underline{x}, \underline{x})$ is negative definite.

Then let us consider the following problems:

$$
\left(P^{\beta}\right)=\left\{\begin{array}{cl}
\text { Maximize } \sum_{t=0}^{+\infty} \beta^{t}\left(u\left(x_{t}, x_{t+1}\right)-u\left(x^{\beta}, x^{\beta}\right)\right) \\
\text { s.t. } \quad \forall t, x_{t+1} \in D\left(x_{t}\right) \\
\\
x_{0} \text { is given } \\
\beta \in\left[\beta_{0}, 1[.\right.
\end{array}\right.
$$


where $\left(x^{\beta}, x^{\beta}\right)$ is the only stationary program for $\left(P_{\beta}\right)$ and

$$
\left(P^{1}\right)=\left\{\begin{aligned}
& \text { Maximize } \sum_{t=0}^{+\infty}\left(u\left(x_{t}, x_{t+1}\right)-u(\underline{x}, \underline{x})\right) \\
& \text { s.t. } \quad \forall t, x_{t+1} \in D\left(x_{t}\right) \\
& x_{0} \text { is given }
\end{aligned}\right.
$$

when $\beta=1$.

Let us denote respectively by $V^{\beta}\left(x_{0}\right)$ and $V^{1}\left(x_{0}\right)$ the value function of problems $\left(P^{\beta}\right)$ and $\left(P^{1}\right)$, that is:

$$
V^{\beta}\left(x_{0}\right)=\max \left\{\sum_{t=0}^{+\infty} \beta^{t}\left(u\left(x_{t}, x_{t+1}\right)-u\left(x^{\beta}, x^{\beta}\right)\right), x_{t+1} \in D\left(x_{t}\right), \forall t\right\}
$$

and

$$
V^{1}\left(x_{0}\right)=\max \left\{\sum_{t=0}^{+\infty}\left(u\left(x_{t}, x_{t+1}\right)-u(\underline{x}, \underline{x})\right), x_{t+1} \in D\left(x_{t}\right), \forall t\right\} \text { when } \beta=1 .
$$

Proposition $6 V^{\beta}$ satisfies the Bellman equation:

$$
V^{\beta}(x)=\max \left\{u(x, y)-u\left(x^{\beta}, x^{\beta}\right)+\beta V^{\beta}(y) ; y \in D(x)\right\} .
$$

Proof: It is quite standard when $\beta<1$. For $\beta=1$, see Dana and Le Van[6].

Dana and Le Van[6] have shown that (H3) implies $\forall x_{0} \geq 0, x_{0} \neq 0$, there exists a good program from $x_{0}$.

For $\beta \in\left[\beta_{0}, 1\right]$, since $u$ is strictly concave, we denote by $g^{\beta}$ the optimal policy, i.e., for any $x \in X, g^{\beta}(x):=\operatorname{argmax}\left\{u(x, y)-u\left(x^{\beta}, x^{\beta}\right)+\beta V^{\beta}(y) ; y \in D(x)\right\}$

Let us introduce two assumptions:

(H8) $\forall x_{0} \geq 0, x_{0} \neq 0,\left(x, g^{1}(x)\right) \in$ intgraph $D$.

(H9) Either $D(0)=\{0\}$ or $D(0)$ contains a strictly positive vector.

Then one has :

Proposition $7 V^{\beta}\left(x_{0}\right)$ is continuous with respect to $\beta$ and $x_{0}$, for $\beta \in\left[\beta_{0}, 1\right]$ and $x \in X$.

Proof: 
1) Let us first show that $V^{\beta}\left(x_{0}\right)$ is upper semi-continuous with respect to $\left(\beta, x_{0}\right)$.

Let $\tilde{x}^{\beta}:=\left(x_{0}, x_{1}^{\beta}, x_{2}^{\beta}, \ldots, x_{t}^{\beta}, \ldots\right)$ be the optimal solution from $x_{0}$ to problem $\left(P_{\beta}\right)$. Then by concavity of $u$, for any $\tilde{x} \in \Pi\left(x_{0}\right)$ :

$$
\forall t, u\left(x^{\beta}, x^{\beta}\right)-u\left(x_{t}, x_{t+1}\right) \geq u_{1}\left(x^{\beta}, x^{\beta}\right)\left(x^{\beta}-x_{t}\right)+u_{2}\left(x^{\beta}, x^{\beta}\right)\left(x^{\beta}-x_{t+1}\right)
$$

Then define $\delta_{\beta}$ by:

$$
u\left(x^{\beta}, x^{\beta}\right)-u\left(x_{t}, x_{t+1}\right)=u_{1}\left(x^{\beta}, x^{\beta}\right)\left(x^{\beta}-x_{t}\right)+u_{2}\left(x^{\beta}, x^{\beta}\right)\left(x^{\beta}-x_{t+1}\right)+\delta_{\beta}\left(x_{t}, x_{t+1}\right) .
$$

The function $\delta_{\beta}$ is non-negative. Then, for any $T$, one has, since $\left(x^{\beta}, x^{\beta}\right)$ satisfies the Euler equation:

$$
\begin{aligned}
\sum_{t=0}^{T} \beta^{t}\left(u\left(x^{\beta}, x^{\beta}\right)-u\left(x_{t}, x_{t+1}\right)\right)= & u_{1}\left(x^{\beta}, x^{\beta}\right)\left(x^{\beta}-x_{0}\right)+\beta^{T} u_{2}\left(x^{\beta}, x^{\beta}\right)\left(x^{\beta}-x_{T+1}\right)+ \\
& \sum_{t=0}^{T} \beta^{t} \delta_{\beta}\left(x_{t}, x_{t+1}\right) .
\end{aligned}
$$

with $\sum_{t=0}^{T} \beta^{t} \delta_{\beta}\left(x_{t}, x_{t+1}\right)$ being continuous with respect to $\beta$ and $\tilde{x}$.

We have, for $T \rightarrow+\infty$, as $\beta<1$ :

$$
\sum_{t=0}^{+\infty} \beta^{t}\left(u\left(x_{t}, x_{t+1}\right)-u\left(x^{\beta}, x^{\beta}\right)\right)=u_{1}\left(x^{\beta}, x^{\beta}\right)\left(x_{0}-x^{\beta}\right)-\lim _{T \rightarrow+\infty} \sum_{t=0}^{T} \beta^{t} \delta_{\beta}\left(x_{t}, x_{t+1}\right) .
$$

The same way, we define $\delta_{1}$ by:

$$
\sum_{t=0}^{T}\left(u\left(x_{t}, x_{t+1}\right)-u(\underline{x}, \underline{x})\right)=u_{1}(\underline{x}, \underline{x})\left(x_{0}-\underline{x}\right)+u_{2}(\underline{x}, \underline{x})\left(x_{T+1}-\underline{x}\right)+\sum_{t=0}^{T}-\delta_{1}\left(x_{t}, x_{t+1}\right) .
$$

Either $\tilde{x}$ is good, and $\lim _{t} x_{t}=\underline{x}$, and one has:

$$
\sum_{t=0}^{+\infty}\left(u\left(x_{t}, x_{t+1}\right)-u(\underline{x}, \underline{x})\right)=u_{1}(\underline{x}, \underline{x})\left(x_{0}-\underline{x}\right)+\lim _{T \rightarrow+\infty} \sum_{t=0}^{T}-\delta_{1}\left(x_{t}, x_{t+1}\right) .
$$

or $\tilde{x}$ is not good, since then $\lim _{T \rightarrow+\infty} \sum_{t=0}^{T}\left(u\left(x_{t}, x_{t+1}\right)-u(\underline{x}, \underline{x})\right)=-\infty$ and $\lim _{T \rightarrow+\infty} u_{1}(\underline{x}, \underline{x})\left(x_{0}-\underline{x}\right)+u_{2}(\underline{x}, \underline{x})\left(x_{T+1}-\underline{x}\right)$ is bounded, (1) still holds.

Then define:

$$
\begin{aligned}
& \Phi(\beta, \tilde{x}):=\sum_{t=0}^{+\infty} \beta^{t}\left(u\left(x_{t}, x_{t+1}\right)-u\left(x^{\beta}, x^{\beta}\right)\right), \\
& \Phi(1, \tilde{x}):=\sum_{t=0}^{+\infty}\left(u\left(x_{t}, x_{t+1}\right)-u(\underline{x}, \underline{x})\right) .
\end{aligned}
$$

Since $\lim _{T \rightarrow+\infty} \sum_{t=0}^{T}-\beta^{t} \delta_{\beta}\left(x_{t}, x_{t+1}\right)$ is the decreasing limit of continuous functions 
with respect to $\beta$ and $\tilde{x}$, it is an upper semi-continuous function with respect to $\beta$ and $\tilde{x}$. Hence, $\Phi(\beta, \tilde{x})$ is also upper semi-continuous (u.s.c.) in $(\beta, \tilde{x})$.

Since $V^{\beta}\left(x_{0}\right)=\operatorname{Max}_{\tilde{x} \in \Gamma\left(x_{0}\right)} \Phi(\beta, \tilde{x})$ and $\Gamma$ is continuous then it follows from Berge's Maximum Theorem that $V^{\beta}\left(x_{0}\right)$ is an u.s.c. function.

2) Let us now show that $V^{\beta}\left(x_{0}\right)$ is lower semi-continuous.

Let us first show that for $x_{0} \geq 0, x_{0} \neq 0, \beta \rightarrow V^{\beta}\left(x_{0}\right)$ is lower semi-continuous in 1.

Let us denote by $\left(x_{t}^{\beta}\right)_{t}$ the optimal solution of $\left(P^{\beta}\right)$, that is:

$$
V^{\beta}\left(x_{0}\right)=\sum_{t=0}^{+\infty} \beta^{t}\left(u\left(x_{t}^{\beta}, x_{t+1}^{\beta}\right)-u\left(x^{\beta}, x^{\beta}\right)\right)
$$

and by $\left(x_{t}^{*}\right)_{t}$ the optimal solution of $\left(P^{1}\right)$, that is:

$$
V^{1}\left(x_{0}\right)=\sum_{t=0}^{+\infty}\left(u\left(x_{t}^{*}, x_{t+1}^{*}\right)-u(\underline{x}, \underline{x})\right)
$$

Then by proposition 6 , as soon as $\Gamma_{G}\left(x_{0}\right) \neq \emptyset$ (which is true by $(\mathbf{H 3})$ ), one has $\lim _{t \rightarrow+\infty} x_{t}^{*}=\underline{x}$.

Moreover, as we have $(\underline{x}, \underline{x}) \in \operatorname{intgraph} D$, there exists $T_{0}$ such that $\forall t \geq$ $T_{0},\left(x_{t}^{*}, \underline{x}\right) \in$ intgraph $D$. Let us then fix $T \geq T_{0}$ and define the sequence $\tilde{x}=\left(x_{0}, x_{1}^{*}, \ldots, x_{T+1}^{*}, \underline{x}, \underline{x}, \ldots\right)$. One has:

$$
\begin{aligned}
V^{\beta}\left(x_{0}\right) & =\sum_{t=0}^{+\infty} \beta^{t}\left(u\left(x_{t}^{\beta}, x_{t+1}^{\beta}\right)-u\left(x^{\beta}, x^{\beta}\right)\right) \\
& \geq \sum_{t=0}^{+\infty} \beta^{t}\left(u\left(x_{t}, x_{t+1}\right)-u\left(x^{\beta}, x^{\beta}\right)\right) \text { since } \tilde{x} \text { feasible for }\left(P_{\beta}\right) \\
& \left.=\sum_{t=0}^{+\infty} \beta^{t}\left(u\left(x_{t}, x_{t+1}\right)-u(\underline{x}, \underline{x})\right)+\sum_{t=0}^{+\infty} \beta^{t}(u(\underline{x}, \underline{x}))-u\left(x^{\beta}, x^{\beta}\right)\right) \\
& \geq \sum_{t=0}^{+\infty} \beta^{t}\left(u\left(x_{t}, x_{t+1}\right)-u(\underline{x}, \underline{x})\right) \text { by definition of } \underline{x} \\
& =\sum_{t=0}^{T} \beta^{t}\left(u\left(x_{t}^{*}, x_{t+1}^{*}\right)-u(\underline{x}, \underline{x})\right)+\beta^{T}\left(u\left(x_{T+1}^{*}, \underline{x}\right)-u(\underline{x}, \underline{x})\right)
\end{aligned}
$$

then one has $\forall T \geq T_{0}, \forall \beta \in(0,1)$ :

$$
V^{\beta}\left(x_{0}\right) \geq \sum_{t=0}^{T} \beta^{t}\left(u\left(x_{t}^{*}, x_{t+1}^{*}\right)-u(\underline{x}, \underline{x})\right)+\beta^{T}\left(u\left(x_{T+1}^{*}, \underline{x}\right)-u(\underline{x}, \underline{x})\right)
$$

Then, for $\beta \rightarrow 1$ :

$$
\liminf _{\beta \rightarrow 1} V^{\beta}\left(x_{0}\right) \geq \sum_{t=0}^{T}\left(u\left(x_{t}^{*}, x_{t+1}^{*}\right)-u(\underline{x}, \underline{x})\right)+\left(u\left(x_{T+1}^{*}, \underline{x}\right)-u(\underline{x}, \underline{x})\right)
$$


Then, for $T \rightarrow+\infty$, as $\lim _{T \rightarrow+\infty} x_{T+1}^{*}=\underline{x}$, one has $\liminf _{\beta \rightarrow 1} V^{\beta}\left(x_{0}\right) \geq V^{1}\left(x_{0}\right)$.

Let us now show that $\forall x_{0} \geq 0, x_{0} \neq 0,(\beta, x) \rightarrow V^{\beta}(x)$ is lower semi-continuous in $\left(1, x_{0}\right)$. Indeed, by $(\mathbf{H} 8)$, one has then $\left(x_{0}, g^{1}\left(x_{0}\right)\right) \in$ intgraph $D$,

Since $\left(x_{0}, g^{1}\left(x_{0}\right)\right) \in$ intgraphD, there exists $\mathcal{V}\left(x_{0}\right)$ a neighborhood in $X$ of $x_{0}$, such that $\forall x_{0}^{\prime} \in \mathcal{V}\left(x_{0}\right),\left(x_{0}^{\prime}, g^{1}\left(x_{0}\right)\right) \in$ intgraphD. Then one has:

$$
V^{\beta}\left(x_{0}^{\prime}\right) \geq u\left(x_{0}^{\prime}, g^{1}\left(x_{0}\right)\right)-u\left(x^{\beta}, x^{\beta}\right)+\beta V^{\beta}\left(g^{1}\left(x_{0}\right)\right)
$$

Then one has:

$$
\begin{aligned}
\liminf _{\substack{\beta \rightarrow 1 \\
x_{0}^{\prime} \rightarrow x_{0}}} V^{\beta}\left(x_{0}^{\prime}\right) & \geq u\left(x_{0}, g^{1}\left(x_{0}\right)\right)-u(\underline{x}, \underline{x})+\liminf _{\beta \rightarrow 1} \beta V^{\beta}\left(g^{1}\left(x_{0}\right)\right) \\
& \geq u\left(x_{0}, g^{1}\left(x_{0}\right)\right)-u(\underline{x}, \underline{x})+V\left(g^{1}\left(x_{0}\right)\right)=V^{1}\left(x_{0}\right)
\end{aligned}
$$

Moreover $(\beta, x) \rightarrow V^{\beta}(x)$ is continuous in $(1,0)$. Indeed, from $(\mathbf{H 9})$, we have two cases:

a) $D(0)=0$.

One has $\limsup _{\substack{\beta \rightarrow 1 \\ x \rightarrow 0}} V^{\beta}(x) \leq V^{1}(0)=-\infty$. Then $\lim _{\substack{\beta \rightarrow 1 \\ x \rightarrow 0}} V^{\beta}(x)=-\infty=V^{1}(0)$.

b) $D(0)$ contains a strictly positive vector.

Then there exists a good program from 0 (see Dana-Le Van[6]). Then the proof is the same as previously (when $x_{0} \neq 0$ ). Observe that in this case $V^{1}(0)>-\infty$.

Proposition 8 The optimal policy $\left(\beta, x_{0}\right) \rightarrow g^{\beta}\left(x_{0}\right)$ is continuous at $\left(1, x_{0}\right)$.

Proof: 1) If $x_{0} \neq 0$. Consider the sequences $x_{n} \rightarrow x_{0}, \beta_{n} \rightarrow 1$.

Since $V^{\beta_{n}}\left(x_{n}\right)$ satisfies the Bellman equation and the maximum is in $g^{\beta_{n}}\left(x_{n}\right)$ :

$$
V^{\beta_{n}}\left(x_{n}\right)=u\left(x_{n}, g^{\beta_{n}}\left(x_{n}\right)\right)-u\left(x^{\beta}, x^{\beta}\right)+\beta_{n} V^{\beta_{n}}\left(x_{n}\right)
$$

For $\beta_{n} \rightarrow 1, x_{n} \rightarrow x_{0}, g^{\beta_{n}}\left(x_{n}\right) \in D\left(x_{n}\right), \forall n$ and since $D$ is continuous, there exists a subsequence, call it again $g^{\beta_{n}}\left(x_{n}\right)$, that converges to $y \in D\left(x_{0}\right)$. Moreover, by proposition 7, $\lim _{\substack{\beta_{n} \rightarrow 1 \\ x_{n} \rightarrow x_{0}}} V^{\beta_{n}}\left(g^{\beta_{n}}\left(x_{n}\right)\right)=V^{1}(y)$. Then, since $u$ is continuous, one has $V^{1}\left(x_{0}\right)=u\left(x_{0}, y\right)-u(\underline{x}, \underline{x})+V^{1}(y)$. And since $u$ is strictly concave, $y$ is unique and one has $y=g^{1}\left(x_{0}\right)$. Hence $\lim _{\substack{\beta_{n} \rightarrow 1 \\ x_{n} \rightarrow x_{0}}}\left(g^{\beta_{n}}\left(x_{n}\right)\right)=g^{1}\left(x_{0}\right)$.

2) Now consider the case $x_{0}=0$.

2a) $D(0)=\{0\}$. 
One has $\limsup _{\substack{\beta \rightarrow 1 \\ x \rightarrow 0}} V^{\beta}(x) \leq V^{1}(0)=-\infty$. Then $\lim _{\substack{\beta \rightarrow 1 \\ x \rightarrow 0}} V^{\beta}(x)=-\infty=V^{1}(0)$. Moreover, it is clear since $D$ is u.s.c. that $\lim _{\substack{\beta \rightarrow 1 \\ x \rightarrow 0}} g^{\beta}(x)=0=g^{1}(0)$.

2b) $D(0)$ contains a strictly positive vector.

Then there exists a good program from 0 and $V^{1}(0)>-\infty$. Apply the proof in case 1$)$.

Hence:

Corollary 1 The optimal policy $\left(\beta, x_{0}\right) \rightarrow g^{\beta}\left(x_{0}\right)$ is continuous in $\left[\beta_{0}, 1\right] \times X$.

Proof: We already know that the optimal policy $\left(\beta, x_{0}\right) \rightarrow g^{\beta}\left(x_{0}\right)$ is continuous in $\left[\beta_{0}, 1[\times X\right.$. Proposition 8 ends the proof.

\section{About the differentiability of the optimal pol- icy}

Definition $5 u$ is said to be $\left(\alpha, \alpha^{\prime}\right)$-concave if $u(x, y)+\frac{1}{2} \alpha\|x\|^{2}+\frac{1}{2} \alpha^{\prime}\|y\|^{2}$ is concave, where $\alpha>0, \alpha^{\prime}>0$.

We now introduce new assumptions:

(H10) $u$ is $\left(\alpha, \alpha^{\prime}\right)$-concave on graphD.

(H11) There exists a good program from any $x_{0} \geq 0$.

Note that (H10) is satisfied if we assume that $u$ is strongly concave (with negative definite Hessian) in the graph of $D$. Venditti [18] gives conditions on the fundamentals to obtain strong concavity of the utility function.

Assumption (H11) ensures that the function $V^{1}$ is real-valued and continuous on $X$ (see Dana and Le Van $[6]$ ).

Remark 1 1. Santos [13] assumes only $(0, \alpha)$ - concavity. Here, to obtain the differentiability of the optimal policy when $\beta=1$, we use also the results given by Montrucchio [12] which requires $(\alpha, 0)$ - concavity. Combining the assumptions in Santos [13] and Montrucchio [12], we assume $\left(\alpha, \alpha^{\prime}\right)-$ concavity.

2. Assumption (H11), a priori, rules out the case $D(0)=\{0\}$. But Assumption (H3) allows us to restrict to the set of $x$ larger than some $x_{0}>0$. 
Since $V^{\beta}(x)$ is continuous in the compact set $\left[\beta_{0}, 1\right] \times X$, we now prove that there exists a sequence of functions $f_{T}^{\beta}$, concave, twice differentiable in $x$, continuous in $(\beta, x)$, which converges uniformly in $(\beta, x)$ to $V^{\beta}(x)$. The following lemma is crucial for the proof of the differentiability of the optimal policy.

Lemma 1 There exists a sequence of concave functions $f_{T}^{\beta}(x), C^{2}$ in $x$, continuous in $(\beta, x)$, converging uniformly in $\left[\beta_{0}, 1\right] \times X$ to $V^{\beta}(x)$ when $T \rightarrow+\infty$.

\section{Proof:}

Let $T$ be a given integer. Let $\beta \in\left[\beta_{0}, 1\right]$. There exists a $C^{2}$ function $h_{T, \beta}$, concave in $x$, such that

$$
\sup _{x \in X}\left|V^{\beta}(x)-h_{T, \beta}(x)\right| \leq \frac{1}{2 T}
$$

(see Boldrin and Montrucchio[5], Lemma 3.1 p.7-8). Since $V^{\beta}(x)$ is continuous (hence, uniformly continuous) in $(\beta, x) \in\left[\beta_{0}, 1\right] \times X$, there exists $\eta$, independent of $\beta$, such that, $\left|\beta^{\prime}-\beta\right|<\eta$ implies

$$
\sup _{x \in X}\left|V^{\beta}(x)-V^{\beta^{\prime}}(x)\right| \leq \frac{1}{2 T} .
$$

Thus, if $B(\beta, \eta)$ denotes the open ball, we have:

$$
\sup _{x \in X} \sup _{\beta^{\prime} \in B(\beta, \eta)}\left|h_{T, \beta}(x)-V^{\beta^{\prime}}(x)\right| \leq \frac{1}{T} .
$$

Let $\left\{B\left(\beta_{i}, \eta\right)\right\}, i=1, \ldots, I$ be a finite covering of $\left[\beta_{0}, 1\right]$. Consider a partition of unity $\left(\varphi_{i}^{T}\right)_{i=1, \ldots, I}$ dominated by $\left\{B\left(\beta_{i}, \eta\right)\right\}$, i.e.

(1) $\varphi_{i}^{T}:\left[\beta_{0}, 1\right] \rightarrow[0,1]$,

(2) $\varphi_{i}^{T}\left(\beta^{\prime}\right)=0$ if $\beta^{\prime} \notin B\left(\beta_{i}, \eta\right)$,

(3) and $\sum_{i=1}^{I} \varphi_{i}^{T}\left(\beta^{\prime}\right)=1, \forall \beta^{\prime} \in\left[\beta_{0}, 1\right]$.

Let

$$
f_{T}^{\beta}(x)=\sum_{i=1}^{I} \varphi_{i}^{T}(\beta) h_{T, \beta_{i}}(x) .
$$

one can easily check that

(1) $f_{T}^{\beta}(x)$ is continuous in $(\beta, x)$, concave, $C^{2}$ in $x$,

(2) and $\sup _{x \in X} \sup _{\beta \in\left[\beta_{0}, 1\right]}\left|V^{\beta}(x)-f_{T}^{\beta}(x)\right| \leq \frac{1}{T}$. 
Consider now the following sequence of optimal finite-horizon programs:

$$
(P 1)_{T}^{\beta}=\left\{\begin{array}{cl}
\operatorname{Max} & \sum_{t=0}^{T-1} \beta^{t}\left(u\left(x_{t}, x_{t+1}\right)-u\left(x^{\beta}, x^{\beta}\right)\right)+\beta^{T} f_{T}^{\beta}\left(x_{T}\right) \\
\text { s.t. } & x_{t+1} \in D\left(x_{t}\right), \forall t=0, \ldots, T-1 \\
& x_{0} \neq 0 \text { is given }, \beta \in\left[\beta_{0}, 1\right] .
\end{array}\right.
$$

Let us call $g_{T}^{\beta}(x)$ the associated policy function, such that the optimal solution of the problem is $\forall t, x_{t}=g_{T}^{\beta, t}(x)$.

Let us also associate with $T$ the sequence of following problems:

$$
\begin{aligned}
(P 2)_{n \leq T}^{\beta}: & V_{0}^{\beta}=f_{T}^{\beta} \\
& \left.V_{n}^{\beta}(x)=\max _{y \in D(x)}\left\{u(x, y)-u\left(x^{\beta}, x^{\beta}\right)\right)+\beta V_{n-1}^{\beta}(y)\right\}, \forall n \leq T
\end{aligned}
$$

Lemma $2 \forall n \leq T$, one has $V_{n}^{\beta}(x)=\max \left\{\sum_{t=0}^{n-1} \beta^{t}\left(u\left(x_{t}, x_{t+1}\right)-u\left(x^{\beta}, x^{\beta}\right)\right)+\right.$ $\left.\beta^{n} f_{T}^{\beta}\left(x_{n}\right) ; x_{t+1} \in D\left(x_{t}\right), \forall t\right\}$.

Proof:

Let us first show that $V_{n}^{\beta}(x) \geq \max \left\{\sum_{t=0}^{n-1} \beta^{t}\left(u\left(x_{t}, x_{t+1}\right)-u\left(x^{\beta}, x^{\beta}\right)\right)+\beta^{n} f_{T}^{\beta}\left(x_{n}\right)\right\}$. Let us consider $\left(x_{1}^{*}, \ldots, x_{n}^{*}\right)$ optimal solution that is $\left(x_{1}^{*}, \ldots, x_{n}^{*}\right)$ is such that

$$
\begin{aligned}
W_{n}\left(x_{0}\right) & :=\sum_{t=0}^{n-1} \beta^{t}\left(u\left(x_{t}^{*}, x_{t+1}^{*}\right)-u\left(x^{\beta}, x^{\beta}\right)\right)+\beta^{n} f_{T}^{\beta}\left(x_{n}^{*}\right) \\
& =\max _{x_{t+1} \in D\left(x_{t}\right), \forall t} \sum_{t=0}^{n-1} \beta^{t}\left(u\left(x_{t}, x_{t+1}\right)-u\left(x^{\beta}, x^{\beta}\right)\right)+\beta^{n} f_{T}^{\beta}\left(x_{n}\right) .
\end{aligned}
$$

Then one has:

$$
\begin{aligned}
W_{n}\left(x_{0}\right) & \leq \sum_{t=0}^{n-2} \beta^{t}\left(u\left(x_{t}^{*}, x_{t+1}^{*}\right)-u\left(x^{\beta}, x^{\beta}\right)\right)+\beta^{n-1} V_{1}^{\beta}\left(x_{n-1}^{*}\right) \\
& \leq \sum_{t=0}^{n-3} \beta^{t}\left(u\left(x_{t}^{*}, x_{t+1}^{*}\right)-u\left(x^{\beta}, x^{\beta}\right)\right)+\beta^{n-2} V_{2}^{\beta}\left(x_{n-2}^{*}\right) \\
& \leq \cdots \leq u\left(x_{0}, x_{1}^{*}\right)+\beta V_{n-1}^{\beta}\left(x_{1}^{*}\right) \leq V_{n}^{\beta}\left(x_{0}\right)
\end{aligned}
$$

Let us now show that $V_{n}^{\beta}(x) \leq \max \left\{\sum_{t=0}^{n-1} \beta^{t}\left(u\left(x_{t}, x_{t+1}\right)-u\left(x^{\beta}, x^{\beta}\right)\right)+\beta^{n} f_{T}^{\beta}\left(x_{n}\right)\right\}$. 
There exists $\left(x_{1}^{*}, \ldots, x_{n}^{*}\right)$ such that :

$$
\begin{aligned}
& \left.V_{n}^{\beta}\left(x_{0}\right)=u\left(x_{0}, x_{1}^{*}\right)-u\left(x^{\beta}, x^{\beta}\right)\right)+\beta V_{n-1}^{\beta}\left(x_{1}^{*}\right) \\
& \left.V_{n-1}^{\beta}\left(x_{1}^{*}\right)=u\left(x_{1}^{*}, x_{2}^{*}\right)-u\left(x^{\beta}, x^{\beta}\right)\right)+\beta V_{n-2}^{\beta}\left(x_{2}^{*}\right) \\
& \left.V_{1}^{\beta}\left(x_{n-1}^{*}\right)=u\left(x_{n-1}, x_{n}^{*}\right)-u\left(x^{\beta}, x^{\beta}\right)\right)+\beta V_{0}^{\beta}\left(x_{n}^{*}\right) \\
& \text { that is } \\
& V_{n}^{\beta}\left(x_{0}\right)=\sum_{t=0}^{n-1} \beta^{t}\left(u\left(x_{t}^{*}, x_{t+1}^{*}\right)-u\left(x^{\beta}, x^{\beta}\right)\right)+\beta^{n} f_{T}^{\beta}\left(x_{n}^{*}\right) \leq W_{n}\left(x_{0}\right)
\end{aligned}
$$

Lemma 3 There exists $\beta_{0}^{\prime} \in\left[\beta_{0}, 1\left[, T_{0}\right.\right.$ such that $\forall \beta \in\left[\beta_{0}^{\prime}, 1\right], \forall T \geq T_{0}$, the optimal path $\left\{g_{T}^{\beta, t}(x)\right\}_{t}$ of problem $(P 1)_{T}^{\beta}$ is interior.

Proof: From (H8), for $\beta$ close enough to one, $\forall x_{0}, g^{\beta}\left(x_{0}\right)$ is interior. Then (see Boldrin and Montrucchio[5], Lemma 3.1 p.7), there exists $\sigma$, such that if $\sup _{\beta, x}\left|f_{T}^{\beta}(x)-V^{\beta}(x)\right|<\sigma$, then $g_{T}^{\beta}\left(x_{0}\right)$ is interior. And if $T f_{T}^{\beta}(x)=\operatorname{Max}\{u(x, y)-$ $\left.u\left(x^{\beta}, x^{\beta}\right)+\beta f_{T}^{\beta}(y) ; y \in D(x)\right\}$, then $\sup _{\beta, x}\left|T f_{T}^{\beta}(x)-V^{\beta}(x)\right|<\sigma$, and one has also $g_{T}^{\beta, 2}(x)$ is interior. And so on by induction, $\forall T,\left\{g_{T}^{\beta, t}(x)\right\}_{t}$ is interior.

Lemma 4 For $\beta \in\left[\beta_{0}^{\prime}, 1\right]$, recall that $g^{\beta}$ is the optimal policy associated with $V^{\beta}$. Let $g^{\beta, t}$ denote the $t-t h$ iterate of $g^{\beta}$. Then, $\forall \beta \in\left[\beta_{0}^{\prime}, 1\right], \forall T, \forall t \leq T$, there exists a constant $k(t)$ such that:

$$
\left\|g^{\beta, t}-g_{T}^{\beta, t}\right\| \leq k(t) \sup _{\beta, x}\left|V^{\beta}(x)-f_{T}^{\beta}(x)\right|^{\frac{1}{2^{t+1}}} .
$$

Thus, $\forall t, g_{T}^{\beta, t}$ converges uniformly with respect to $(\beta, x)$ to $g^{\beta, t}$ when $f_{T}^{\beta}$ converges to $V^{\beta}$.

Proof: We show this by induction. Indeed, one has by lemma 2:

$$
\begin{aligned}
V_{T}^{\beta}\left(x_{0}\right) & =\max \left\{\sum_{t=0}^{T-1} \beta^{t}\left(u\left(x_{t}, x_{t+1}\right)-u\left(x^{\beta}, x^{\beta}\right)\right)+\beta^{T} f_{T}^{\beta}\left(x_{T}\right) ; x_{t+1} \in D\left(x_{t}\right), \forall t\right\} \\
& =\sum_{t=0}^{T-1} \beta^{t}\left(u\left(g_{T}^{\beta, t}\left(x_{0}\right), g_{T}^{\beta, t+1}\left(x_{0}\right)\right)-u\left(x^{\beta}, x^{\beta}\right)\right)+\beta^{T} f_{T}^{\beta}\left(g_{T}^{\beta, T}\left(x_{0}\right)\right) \\
& =\max \left\{u\left(x_{0}, y\right)-u\left(x^{\beta}, x^{\beta}\right)+\beta V_{T-1}^{\beta}(y) ; y \in D\left(x_{0}\right)\right\} \\
& =u\left(x_{0}, g_{T}^{\beta}\left(x_{0}\right)\right)-u\left(x^{\beta}, x^{\beta}\right)+\beta V_{T-1}^{\beta}\left(g_{T}^{\beta}\left(x_{0}\right)\right)
\end{aligned}
$$

where

$$
g_{T}^{\beta}(x)=\operatorname{argmax}\left\{u(x, y)-u\left(x^{\beta}, x^{\beta}\right)+\beta V_{T-1}^{\beta}(y)\right\}
$$


In the following, \|\| will denote the sup-norm with respect to $x$. From $(\mathbf{H 1 0})$, $V^{\beta}$ is $(\alpha, 0)$-concave. Montrucchio's Theorem 2 [12] applies and one has:

$$
\begin{gathered}
\left\|V_{T-1}^{\beta}-V^{\beta}\right\| \leq\left\|V^{\beta}-f_{T}^{\beta}\right\| \leq\left\|V^{\beta}-f_{T}^{\beta}\right\|^{\frac{1}{2}} \text { (for } T \text { big enough). } \\
\left\|g^{\beta}-g_{T}^{\beta}\right\| \leq 2 \alpha^{-1}\left\|V^{\beta}-f_{T}^{\beta}\right\|^{\frac{1}{2}},
\end{gathered}
$$

where

$$
g^{\beta}(x)=\operatorname{argmax}\left\{u(x, y)-u\left(x^{\beta}, x^{\beta}\right)+\beta V^{\beta}(y)\right\} .
$$

Let

$$
\begin{gathered}
g^{\beta, 2}(x)=\operatorname{argmax}\left\{u\left(g^{\beta}(x), y\right)-u\left(x^{\beta}, x^{\beta}\right)+\beta V^{\beta}(y)\right\} \\
g_{T}^{\beta, 2}(x)=\operatorname{argmax}\left\{u\left(g_{T}^{\beta}(x), y\right)-u\left(x^{\beta}, x^{\beta}\right)+\beta V_{T-2}^{\beta}(y)\right\}
\end{gathered}
$$

Since $u$ is $C^{1}$, there exists a constant $a$ independent of $x$ and $T$ such that:

$$
\begin{aligned}
\left\|u\left(g^{\beta}(x), y\right)+\beta V^{\beta}(y)-u\left(g_{T}^{\beta}(x), y\right)-\beta V_{T-2}^{\beta}(y)\right\| & \leq a\left\|g^{\beta}-g_{T}^{\beta}\right\|+\left\|V^{\beta}-V_{T-2}^{\beta}\right\| \\
& \leq\left(2 a \alpha^{-1}+1\right)\left\|V^{\beta}-f_{T}^{\beta}\right\|^{\frac{1}{2}},
\end{aligned}
$$

since Theorem $2[12]$ implies that $\left\|V^{\beta}-V_{T-2}^{\beta}\right\| \leq\left\|V^{\beta}-f_{T}\right\|$. Then by Lemma 2 [12], one obtains:

$\left\|g^{\beta, 2}-g_{T}^{\beta, 2}\right\| \leq 2 \alpha^{-1}\left(2 a \alpha^{-1}+1\right)^{\frac{1}{2}}\left\|V^{\beta}-f_{T}\right\|^{\frac{1}{2}}=k(2)\left\|V^{\beta}-f_{T}\right\|^{\frac{1}{4}} \leq k(2) \sup _{\beta, x}\left|V^{\beta}(x)-f_{T}^{\beta}(x)\right|$.

and so on.

Proposition $9 g_{T}^{\beta}(x)$ converges uniformly to $g^{\beta}(x)$ with respect to $(\beta, x)$, in $\left[\beta_{0}^{\prime}, 1\right] \times X$, and $g_{T}^{\beta}(x)$ is $C^{1}$.

Proof: That $g_{T}^{\beta}\left(x_{0}\right)$ converges uniformly to $g^{\beta}\left(x_{0}\right)$ with respect to $(\beta, x)$ is an immediate corollary of the previous lemma.

We now show that the functions $V_{t}^{\beta}$ are $C^{2}$ in $x$ for $t=1, \ldots, T$. First, we have:

$$
V_{1}^{\beta}(x)=\max \left\{u(x, y)-u\left(x^{\beta}, x^{\beta}\right)+\beta f_{T}^{\beta}(y) ; y \in D(x)\right\} .
$$

Let $\zeta_{T}^{\beta}(x)$ denote the argmax of this problem, i.e.:

$$
V_{1}^{\beta}(x)=u\left(x, \zeta_{T}^{\beta}(x)\right)-u\left(x^{\beta}, x^{\beta}\right)+\beta f_{T}^{\beta}\left(\zeta_{T}^{\beta}(x)\right) .
$$

Then $\zeta_{T}^{\beta}(x)$ satisfies:

$$
u_{2}\left(x, \zeta_{T}^{\beta}(x)\right)+\beta\left(f_{T}^{\beta}\right)^{\prime}\left(\zeta_{T}^{\beta}(x)\right)=0 .
$$


Since $f_{T}^{\beta}$ is $C^{2}$, concave and $u$ is $\left(0, \alpha^{\prime}\right)$-concave, the function $\zeta^{\beta}$ is $C^{1}$ and thus, $V_{1}^{\beta}$ is $C^{2}$. By induction, $V_{T-1}^{\beta}$ is $C^{2}$.

Since $g_{T}^{\beta}$ is defined by:

$$
u\left(x, g_{T}^{\beta}(x)\right)+\beta V_{T-1}^{\beta}\left(g_{T}^{\beta}(x)\right)=\max _{y \in D(x)}\left\{u(x, y)+\beta V_{T-1}^{\beta}(y)\right\},
$$

then $g_{T}^{\beta}(x)$ must satisfy:

$$
u_{2}\left(x, g_{T}^{\beta}(x)\right)+\beta\left(V_{T-1}^{\beta}\right)^{\prime}\left(g_{T}^{\beta}(x)\right)=0 .
$$

That $(\beta, x) \rightarrow g_{T}^{\beta}(x)$ is $C^{1}$ follows then from the implicit function theorem, since $V_{T-1}^{\beta}$ is $C^{2}$, concave and $u$ is $\left(0, \alpha^{\prime}\right)$-concave. Obviously, $V_{T}^{\beta}$ is $C^{2}$.

We now prove that $D g_{T}^{\beta}(x)$ converges uniformly. Let us now show that the sequence of derivatives of the policy function of the finite-horizon problems is defined by the policy functions of a sequence of finite-horizon quadratic problems as introduced in Santos[13]. Indeed, $\forall \beta \in\left[\beta_{0}^{\prime}, 1\right]$, let us consider $\tilde{x}$ an optimal solution for the finite-horizon optimization problem $(P 1)_{T}^{\beta}$. By lemma 3, it is interior. Then let us consider the following sequence of finite-horizon quadratic problems:

$$
\left(Q_{T}^{\beta}\right)=\left\{\begin{aligned}
& \text { Maximize } \sum_{t=0}^{T-1}\left[\frac{1}{2} \beta^{t}\left(b_{t}, b_{t+1}\right)^{\prime} \cdot D^{2} u\left(x_{t}, x_{t+1}\right) \cdot\left(b_{t}, b_{t+1}\right)\right] \\
&+\beta^{T}\left[\frac{1}{2} b_{T}^{\prime} \cdot D^{2} f_{T}^{\beta}\left(x_{T}\right) \cdot b_{T}\right] \\
& \text { s.t. } b_{0}=a_{0} \text { is fixed }
\end{aligned}\right.
$$

Lemma 5 If $\left(x_{t}, x_{t+1}\right)$ is an interior solution for the finite-horizon optimization problem $(P 1)_{T}^{\beta}$, then the sequence of vectors $\left\{a_{t}\right\}_{t=0}^{T}$ defined by $a_{t}=D g_{T}^{\beta, t}\left(x_{0}\right) \cdot a_{0}$ is an optimal solution to the problem $\left(Q_{T}^{\beta}\right)$.

Proof: The proof given by Santos[13] applies. Observe that we have the first order conditions:

$$
\begin{gathered}
\forall t=1, \ldots, T-1, \\
D_{21} u\left(x_{t-1}, x_{t}\right) \cdot a_{t-1}+\left[D_{22} u\left(x_{t-1}, x_{t}\right)+\beta D_{11} u\left(x_{t}, x_{t+1}\right)\right] \cdot a_{t}+\beta D_{12} u\left(x_{t}, x_{t+1}\right) \cdot a_{t+1}=0, \\
D_{21} u\left(x_{T-1}, x_{T}\right) \cdot a_{T-1}+\left[D_{22} u\left(x_{T-1}, x_{T}\right)+\beta D^{2} f_{T}^{\beta}\left(x_{T}\right)\right] \cdot a_{T}=0 .
\end{gathered}
$$


Lemma 6 Let $a_{0}$ satisfy $\left\|a_{0}\right\|=1$. Then there exists a constant $M>0$ such that for any $x_{0}$ in $X$, for all optimal sequences $\left\{x_{t}\right\}_{t=0}^{T}$ from $x_{0}$, for any sequence $\left\{a_{t}\right\}_{t=0}^{T}$ defined as in lemma 5 , for any $\beta$ in $\left[\beta_{0}^{\prime}, 1\right]$, for any $T$, one has:

$$
\sum_{t=0}^{T-1} \beta^{t}\left[\frac{1}{2}\left(a_{t}, a_{t+1}\right)^{\prime} \cdot D^{2} u\left(x_{t}, x_{t+1}\right) \cdot\left(a_{t}, a_{t+1}\right)\right] \geq-M .
$$

Moreover, one has that $\left\|a_{1}\right\| \leq\left(\frac{2 M}{\alpha^{\prime}}\right)^{\frac{1}{2}}$.

Proof: The proof given by Santos[13] applies. It relies on the $\left(\alpha, \alpha^{\prime}\right)$-concavity of $u$.

CONDITION $D^{\beta}$ : Let us consider an optimal solution to the infinite-horizon problem $\left(P^{\beta}\right), \beta \in\left[\beta_{0}^{\prime}, 1\right]$. Then a sequence of vectors $\left\{a_{t}\right\}_{t}$ with $\left\|a_{0}\right\|=1$ is said to satisfy Condition $D^{\beta}$ if:

$$
\begin{gathered}
\left(D^{\beta} 1\right) \quad \forall t \geq 1, D_{21} u\left(x_{t-1}, x_{t}\right) \cdot a_{t-1}+\left[D_{22} u\left(x_{t-1}, x_{t}\right)+\beta D_{11} u\left(x_{t}, x_{t+1}\right)\right] \cdot a_{t} \\
+\beta D_{12} u\left(x_{t}, x_{t+1}\right) \cdot a_{t+1}=0 . \\
(D 2) \quad \begin{array}{c}
\exists M>0 \text { such that } \forall \beta \in\left[\beta_{0}^{\prime}, 1\right], \\
\end{array} \\
\quad \sum_{t=0}^{+\infty} \beta^{t}\left[\frac{1}{2}\left(a_{t}, a_{t+1}\right)^{\prime} \cdot D^{2} u\left(x_{t}, x_{t+1}\right) \cdot\left(a_{t}, a_{t+1}\right)\right] \geq-M .
\end{gathered}
$$

Remark 2 (D $\left.D^{\beta} 1\right)$ corresponds to the first-order necessary condition of the quadratic optimization problem, while (D2) implies $\forall \beta \in\left[\beta_{0}^{\prime}, 1\right]$ that $\sum_{t=0}^{+\infty} \beta^{t}\left\|a_{t+1}\right\|^{2} \leq \frac{M}{\alpha^{\prime}}$. Indeed, by (H10), $D^{2} u+2 \alpha\left(\begin{array}{cc}I & \\ & 0\end{array}\right)+2 \alpha^{\prime}\left(\begin{array}{cc}I & \\ & 0\end{array}\right) \leq 0$, then (D2) implies $\sum_{t=0}^{+\infty}-\frac{1}{2} \beta^{t}\left(a_{t}, a_{t+1}\right)^{\prime} \cdot\left[-2 \alpha\left(\begin{array}{cc}I & \\ & 0\end{array}\right)-2 \alpha^{\prime}\left(\begin{array}{cc}I & \\ & 0\end{array}\right)\right] .\left(a_{t}, a_{t+1}\right) \leq M$, and $\sum_{t=0}^{+\infty} \beta^{t} \alpha\left\|a_{t}\right\|^{2}+\beta^{t} \alpha^{\prime}\left\|a_{t+1}\right\|^{2} \leq M$ and $\sum_{t=0}^{+\infty} \beta^{t}\left\|a_{t+1}\right\|^{2} \leq \frac{M}{\alpha^{\prime}}$.

Lemma 7 Let $\left\{x_{t}\right\}_{t \geq 0}$ be an interior optimal solution to the infinite-horizon problem $\left(P^{\beta}\right)$. Then a sequence of vectors $\left\{a_{t}\right\}_{t \geq 0}$ satisfies Condition $D^{\beta}$ if and only if it is an optimal solution to the quadratic optimization problem:

$$
\left(Q_{\infty}^{\beta}\right)=\left\{\begin{array}{l}
\text { Maximize } \sum_{t=0}^{+\infty} \beta^{t}\left[\frac{1}{2}\left(b_{t}, b_{t+1}\right)^{\prime} \cdot D^{2} u\left(x_{t}, x_{t+1}\right) \cdot\left(b_{t}, b_{t+1}\right)\right] \\
\text { s.t. } b_{0}=a_{0} \text { is fixed },\left\|a_{0}\right\|=1 .
\end{array}\right.
$$


Proof: The proof given by Santos[13] applies.

Lemma 8 Let $\left\{x_{t}\right\}_{t}$ be an interior optimal solution. Then the optimal solution to the quadratic infinite-horizon problem $\left(Q_{\infty}^{\beta}\right)$ exists and is unique.

\section{Proof:}

As long as the objective is strictly concave, if there exists a solution, it is unique.

Let us prove the existence of a solution.

Define the following finite-horizon quadratic program:

$$
\left(R_{T}^{\beta}\right)=\left\{\begin{array}{ccc}
\operatorname{Maximize} & \sum_{t=0}^{T-1} \beta^{t}\left[\frac{1}{2}\left(b_{t}^{T}, b_{t+1}^{T}\right)^{\prime} D^{2} u\left(x_{t}, x_{t+1}\right)\left(b_{t}^{T}, b_{t+1}^{T}\right)\right] \\
\left\{b_{t}^{T}\right\}_{t=0}^{T} & & \\
& \text { s.t. } \quad & b_{0}^{T}=a_{0} \text { is given } \\
& \beta \in\left[\beta_{0}^{\prime}, 1\right] .
\end{array}\right.
$$

and the following set:

$$
G_{T}^{\beta}=\left\{\begin{aligned}
& \left(b_{0}^{T}, \ldots, b_{T}^{T}\right) \in \mathbb{R}^{(T+1) n} \text { s.t. } b_{0}^{T}=a_{0} \text { and } \\
& \left.\sum_{t=0}^{T-1} \beta^{t}\left[\frac{1}{2}\left(b_{t}^{T}, b_{t+1}^{T}\right)^{\prime} D^{2} u\left(x_{t}, x_{t+1}\right)\left(b_{t}^{T}, b_{t+1}^{T}\right)\right] \geq-M\right\}
\end{aligned}\right.
$$

Assumption (H10) implies that $\forall \beta \in\left[\beta_{0}^{\prime}, 1\right], G_{T}^{\beta}$ is a compact set. Then the problem $\left(R_{T}^{\beta}\right)$ has a unique optimal solution, let us call it $\left\{a_{t}^{T}\right\}_{t=0}^{T}$. This sequence must satisfy the equation: $\forall t=1, \ldots, T$,

$$
\begin{array}{r}
D_{21} u\left(x_{t-1}, x_{t}\right) \cdot a_{t-1}^{T}+\left[D_{22} u\left(x_{t-1}, x_{t}\right)+\beta D_{11} u\left(x_{t}, x_{t+1}\right)\right] \cdot a_{t}^{T} \\
+\beta D_{12} u\left(x_{t}, x_{t+1}\right) \cdot a_{t+1}^{T}=0 .
\end{array}
$$

Since $\forall T, \tilde{a}_{T}=\left\{a_{t}^{T}\right\}_{t=0}^{T} \in G_{T}^{\beta}$ and $u$ is $\left(0, \alpha^{\prime}\right)$-concave, there exists a uniform constant $N_{t}$ such that $\forall T,\left\|a_{t}^{T}\right\| \leq N_{t}$. Then, $\forall t,\left(a_{t}^{T}\right)_{T}$ belongs to a compact set, and there exists a subsequence of $\left\{a_{t}^{T}\right\}_{t=0}^{T}$ that converges for the product topology as $T \rightarrow+\infty$. Since $\left\{a_{t}^{T}\right\}_{t=0}^{T} \in G_{T}^{\beta}, \forall T$ and by (1), one has that this limit, call it $\left\{a_{t}\right\}_{t}$, satisfies condition $D^{\beta}$. Then by lemma $7,\left\{a_{t}\right\}_{t}$ is optimal for $\left(Q_{\infty}^{\beta}\right)$.

Now define the sets:

$$
L\left(\beta, x_{0}\right)= \begin{cases} & \left(a_{0}, a_{1}\right) \in \mathbb{R}^{2 n} \text { with }\left\|a_{0}\right\|=1 \text { s.t. } \\ & \left.\exists\left\{a_{t}\right\}_{t \geq 0} \text { which satisfies Condition } D^{\beta}\right\}\end{cases}
$$


Lemma 8 implies that $\forall a_{0}$, there exists a unique $a_{1}$ which satisfies this property. That is, $L\left(\beta, x_{0}\right)$ is the graph of a linear function restricted to the unit sphere. The goal is now to show that $L\left(\beta, x_{0}\right)$ is the graph of the derivative of $g$ at $x_{0}$.

Lemma 9 The correspondence $L$ is continuous and compact-valued in $\left[\beta_{0}^{\prime}, 1\right] \times U$.

Proof: The proof given by Santos[13] applies. See Appendix.

Lemma 10 The sequence of derivative functions $\left\{D g_{T}^{\beta}(x)\right\}_{T \geq 1}$ converges uniformly with respect to $(\beta, x)$.

Proof: The proof is the same as in Santos[13]. The idea is to show that the set

$$
G_{T}^{\beta}\left(x_{0}\right)=\left\{\left(a_{0}, a_{1}\right) \in \mathbb{R}^{2 n} \text { with }\left\|a_{0}\right\|=1 \text { s.t. } a_{1}=D g_{T}^{\beta}\left(x_{0}\right) \cdot a_{0}\right\}
$$

converge uniformly in $\left(\beta, x_{0}\right)$ to $L\left(\beta, x_{0}\right)$ when $T \rightarrow+\infty$. Hence, by taking $a_{0}$ successively equal to the unit-vectors of the basis of $\mathbb{R}^{n}$, we obtain that the partial derivatives of $g^{\beta}$ exist and are continuous with respect to $\left(\beta, x_{0}\right)$. For more details of the proof, see the appendix.

To sum up, we have proved:

Theorem 1 The optimal policy $g^{\beta}$ is differentiable in $X$ for any $\beta$ in $\left[\beta_{0}^{\prime}, 1\right]$. Moreover, the derivative $D g^{\beta}$ is continuous with respect to $(\beta, x)$ in $\left[\beta_{0}^{\prime}, 1\right] \times X$.

Remark 3 Santos[13] assumes that $u$ is $\left(0, \alpha^{\prime}\right)$-concave and that the secondorder derivatives of $u$ are uniformly bounded along every optimal path. Montrucchio[12] assumes that $\left\|D_{11}^{2}\right\|$ and $\left\|D_{12}^{2}\right\|$ are bounded and another condition which is satisfied by $(\alpha, 0)$-concavity. It is straightforward to check that their conditions are satisfied if $u$ is $C^{2}$ and $\left(\alpha, \alpha^{\prime}\right)$ - concave on graphD.

\section{Remark 4 About the convergence of optimal paths to the steady state}

We show that the differentiability of the policy function allows us to obtain easily the turnpike property near 1. Here, the turnpike result becomes a by-product of the differentiability of the policy function.

Claim 1 (The Visit Lemma) Let $\hat{x}$ be expansible. Then we have: $\forall \varepsilon>0, \exists \beta(\varepsilon)>$ 0 such that $\forall x_{0} \geq \hat{x}, \forall \beta \in[\beta(\varepsilon), 1], \exists \bar{t},\left\|g^{\beta, \bar{t}}\left(x_{0}\right)-x^{\beta}\right\|<\varepsilon$. 
Proof: See Scheinkman[17].

Claim $2\left\|D g^{1}(\underline{x})\right\|<1$.

Proof: We know that when $\beta=1$, the optimal path $\left\{g^{1, t}\left(x_{0}\right)\right\}_{t}$, for any $x_{0}>>0$, converges to the steady state $\underline{x}$. Let $\left\|D g^{1}(\underline{x})\right\|$ denote the largest modulus of the eigenvalues of $D g^{1}(\underline{x})$. We must have $\left\|D g^{1}(\underline{x})\right\| \leq 1$. But actually $\left\|D g^{1}(\underline{x})\right\|<1$. Indeed, let $c_{0}$ be a non-null eigenvector associated with the largest eigenvalue in modulus $\lambda$. Write $c_{0}=a_{0}+i b_{0}$, where $a_{0}$ and $b_{0}$ are the real and imaginary parts of $c_{0}$ and $i^{2}=-1$. Let $a_{t}:=D g^{1, t}(\underline{x}) \cdot a_{0}$ and $b_{t}:=D g^{1, t}(\underline{x}) \cdot b_{0}$. Then we have (see the proof of Theorem 2.1 in Santos[13]):

$-\sum_{t=0}^{+\infty}\left(a_{t}, a_{t+1}\right)^{\prime} \cdot D^{2} u(\underline{x}, \underline{x}) \cdot\left(a_{t}, a_{t+1}\right) \leq M\left\|a_{0}\right\|$ and

$-\sum_{t=0}^{+\infty}\left(b_{t}, b_{t+1}\right)^{\prime} \cdot D^{2} u(\underline{x}, \underline{x}) \cdot\left(b_{t}, b_{t+1}\right) \leq M\left\|b_{0}\right\|$. Then it follows that:

$-\sum_{t=0}^{T}\left(a_{t}-i b_{t}, a_{t+1}-i b_{t+1}\right)^{\prime} \cdot D^{2} u(\underline{x}, \underline{x}) \cdot\left(a_{t}+i b_{t}, a_{t+1}+i b_{t+1}\right) \leq M\left(\left\|a_{0}\right\|+\left\|b_{0}\right\|\right)$.

But, $a_{t}+i b_{t}=D g^{1, t}(\underline{x}) \cdot c_{0}=\lambda^{t} c_{0}$, and $a_{t}-i b_{t}=D g^{1, t}(\underline{x}) \cdot \bar{c}_{0}=\bar{\lambda}^{t} \bar{c}_{0}$, and thus $-\left(\bar{c}_{0}, \bar{\lambda} \bar{c}_{0}\right)^{\prime} \cdot D^{2} u(\underline{x}, \underline{x}) \cdot\left(c_{0}, \lambda c_{0}\right) \sum_{t=0}^{+\infty}|\lambda|^{2 t} \leq M\left(\left\|a_{0}\right\|+\left\|b_{0}\right\|\right)$, which implies $|\lambda|<1$.

Claim 3 Let $\hat{x}$ be expansible. Then there exists $\beta_{1}$ such that $\forall x_{0} \geq \hat{x}, \forall \beta \in$ $\left[\beta_{1}, 1\right], \lim _{t \rightarrow+\infty} g^{\beta, t}\left(x_{0}\right)=x^{\beta}$.

Proof: For $\beta=1$, we know by proposition 1 that $\lim _{t} x_{t}=\underline{x}$, and by lemma 2 that $\left\|D g^{1}(\underline{x})\right\|<\xi<1$. Since $(\beta, x) \rightarrow D g^{\beta}(x)$ exists and is continuous with respect to $(\beta, x),\left\|D g^{\beta}(x)\right\|$ is continuous with respect to $(\beta, x)$ and there exists $\beta_{2}^{\prime}$ such that for any $\beta \in\left[\beta_{2}^{\prime}, 1\right],\left\|D g^{\beta}\left(x^{\beta}\right)\right\|<\xi<1$. Then consider $\eta>0$ such that $\xi+\eta<1$. Since $\left\|D g^{\beta}(x)\right\|$ is continuous with respect to $(\beta, x)$ in the compact set $\left[\beta_{2}^{\prime}, 1\right] \times X$, it is uniformly continuous with respect to $(\beta, x)$ and there exists $\varepsilon>0$ such that:

$$
\forall \beta, \beta^{\prime} \in\left[\beta_{2}^{\prime}, 1\right],\left|\beta-\beta^{\prime}\right|<\varepsilon,\left\|x_{0}-x^{\beta}\right\|<\varepsilon \Rightarrow\left|\left\|D g^{\beta^{\prime}}\left(x_{0}\right)\right\|-\left\|D g^{\beta}\left(x^{\beta}\right)\right\|\right| \leq \eta .
$$

In particular (take $\left.\beta=\beta^{\prime}\right)$ : $\left\|x_{0}-x^{\beta}\right\|<\varepsilon \Rightarrow\left\|D g^{\beta}\left(x_{0}\right)\right\| \leq \eta+\xi<1$. That is, by claim $2, \exists \varepsilon>0$ such that $\forall \beta \in\left[\beta_{1}, 1\right],\left\|x_{0}-x^{\beta}\right\|<\varepsilon \Rightarrow \lim _{t \rightarrow+\infty} g^{\beta, t}\left(x_{0}\right)=x^{\beta}$.

Remark 5 1. Scheinkman[17] and McKenzie[9] only assume that the Hessian of $u$ is negative definite at $(\underline{x}, \underline{x})$. But they did not prove that the optimal policy is differentiable. 
2. We obtain the turnpike result without assuming, as in McKenzie[9], that $\operatorname{det} D u_{12}(\underline{x}, \underline{x}) \neq 0$ contrary to Scheinkman[17]. 


\section{Appendix}

\subsection{Proof of lemma 9}

We extend here the proof of lemma 9 given by Santos[13]:

1) Let us first show that $L$ is u.s.c. and compact-valued.

Let $\left(\beta^{n}, x_{0}^{n}\right)_{n}$ be a sequence that converges to $\left(\beta, x_{0}\right) \in\left[\beta_{0}, 1\right] \times U$.

As $(\beta, x) \rightarrow g^{\beta}(x)$ is continuous on $\left[\beta_{0}, 1\right] \times U$, one has that $g^{\beta^{n}}\left(x_{0}^{n}\right) \rightarrow g^{\beta}\left(x_{0}\right)$. By lemma 8 , for the optimal sequence $\left\{x_{t}^{n}\right\}_{t \geq 0}$ where $x_{t}^{n}=g^{\beta^{n}, t}\left(x_{0}^{n}\right)$, there exists $\left\{a_{t}^{n}\right\}_{t \geq 0}$ optimal solution to:

$$
\left(R_{\infty}^{\beta^{n}}\right)=\left\{\begin{array}{cc}
\text { Maximize } & \sum_{t=0}^{+\infty}\left(\beta^{n}\right)^{t}\left[\frac{1}{2}\left(b_{t}^{n}, b_{t+1}^{n}\right)^{\prime} D^{2} u\left(x_{t}^{n}, x_{t+1}^{n}\right)\left(b_{t}^{n}, b_{t+1}^{n}\right)\right] \\
\left\{b_{t}^{n}\right\}_{t \geq 0} & \\
& \text { s.t. } \quad b_{0}^{n}=a_{0}^{n} \text { is given } \\
& \beta^{n} \in\left[\beta_{0}, 1\right] .
\end{array}\right.
$$

One has $\forall n,\left\{a_{t}^{n}\right\}_{t=0}^{\infty}$ satisfies Condition $D^{\beta^{n}}$ (with $\left(x_{t}^{n}\right)_{t}$ ). Since the constant $M$ is independent of $n$, then, since $u$ is $\left(0, \alpha^{\prime}\right)$-concave, $\forall t, \exists N_{t}>0$ such that $\forall n,\left\|a_{t}^{n}\right\| \leq N_{t}$. Then, since the sequence belongs to a compact set of the product topology, there exists a subsequence of $\left(\left\{a_{t}^{n}\right\}_{t=0}^{\infty}\right)_{n}$ that converges, say to $\left\{a_{t}\right\}_{t=0}^{\infty}$. As in the previous lemma, since $\beta^{n} \rightarrow \beta$ and $x_{0}^{n} \rightarrow x_{0}$, one has, since the optimal policy is continuous, $x_{t}^{n} \rightarrow x_{t}$, and hence $\left\{a_{t}\right\}_{t=0}^{\infty}$ satisfies condition $D^{\beta}$ and is then optimal. Hence $\left(\beta^{n}, x_{0}^{n}\right)_{n}$ converges to $\left(\beta, x_{0}\right)$ and $\left(a_{0}^{n}, a_{1}^{n}\right)_{n}$ converges to $\left(a_{0}, a_{1}\right) \in L\left(\beta, x_{0}\right)$, with $\left(a_{0}^{n}, a_{1}^{n}\right) \in L\left(\beta^{n}, x_{0}^{n}\right)$ : that is $L$ is u.s.c. Moreover, since one also has $\left\|a_{1}\right\| \leq\left(\frac{2 M}{\alpha}\right)^{\frac{1}{2}}$ (cf lemma 6$), L\left(\beta, x_{0}\right)$ is a compact set.

2) Let us now show that $L$ is l.s.c.

Pick $\left(a_{0}, a_{1}\right) \in L\left(\beta, x_{0}\right)$ and assume that $\left(\beta^{n}, x_{0}^{n}\right) \rightarrow\left(\beta, x_{0}\right)$. Take $\left(a_{0}^{n}, a_{1}^{n}\right)$ with $a_{0}^{n}=a_{0}$ and $\left(a_{0}^{n}, a_{1}^{n}\right) \in L\left(\beta^{n}, x_{0}^{n}\right), \forall n$. Then the corresponding optimal sequence $\left(\left\{a_{t}^{n}\right\}_{t=0}^{\infty}\right)_{n}$ has a subsequence that converges to, say, $\left\{a_{t}\right\}_{t=0}^{\infty}$. In fact, every subsequence converges to this limit, or else $\left(Q_{\infty}^{\beta}\right)$ would have several solutions, which is impossible since the objective is strictly concave. That is $\left(a_{0}^{n}, a_{1}^{n}\right) \rightarrow\left(a_{0}, a_{1}\right)$. 


\subsection{Proof of lemma 10}

By proposition $9,\left\{g_{T}\right\}_{T \geq 1}$ converges uniformly to $g$ on $\left[\beta_{0}, 1\right] \times X$ and $\forall T, D g_{T}$ is well-defined on $\left[\beta_{0}, 1\right] \times X$.

$\operatorname{Pick}\left(\beta, x_{0}\right) \in\left[\beta_{0}, 1\right] \times X$. Define $x_{t}:=g^{\beta, t}\left(x_{0}\right), \forall t$ and let $L_{T}\left(\beta, x_{0}\right)$ be defined by:

$$
\begin{aligned}
L_{T}\left(\beta, x_{0}\right)= & \left\{\left(a_{0}, a_{1}\right) \in \mathbb{R}^{2 n} \text { with }\left\|a_{0}\right\|=1 \text { s.t. } \exists\left\{a_{t}^{T}\right\}_{t \geq 0}\right. \text { which satisfies: } \\
& D_{21} u\left(x_{t-1}, x_{t}\right) \cdot a_{t-1}^{T}+\left[D_{22} u\left(x_{t-1}, x_{t}\right)+\beta D_{11} u\left(x_{t}, x_{t+1}\right)\right] \cdot a_{t}^{T}+ \\
& \beta D_{12} u\left(x_{t}, x_{t+1}\right) \cdot a_{t+1}^{T}=0, \forall t=1, \ldots, T-1 . \\
& \sum_{t=0}^{T-1} \beta^{t}\left[\frac{1}{2}\left(a_{t}^{T}, a_{t+1}^{T}\right)^{\prime} D^{2} u\left(x_{t}, x_{t+1}\right)\left(a_{t}^{T}, a_{t+1}^{T}\right) \geq-M\right\}
\end{aligned}
$$

It is clear that $\forall T, L_{T+1}\left(\beta, x_{0}\right) \subset L_{T}\left(\beta, x_{0}\right)$ and that, as in lemma $9, \forall T, L_{T}\left(\beta, x_{0}\right)$ is u.s.c.. Moreover, $L\left(\beta, x_{0}\right)=\bigcap_{T \geq 1} L_{T}\left(\beta, x_{0}\right)$. Then, $\forall\left(\beta, x_{0}\right) \in\left[\beta_{0}, 1\right] \times X$,

$\forall \varepsilon>0, \exists T_{0}$ such that $\forall T \geq T_{0}, d\left(L_{T}\left(\beta, x_{0}\right), L\left(\beta, x_{0}\right)\right)<\frac{\varepsilon}{9}$, where $d$ denotes the Hausdorff distance.

(i) Let us first show that $L_{T}\left(\beta, x_{0}\right)$ converges uniformly to $L\left(\beta, x_{0}\right)$.

$L\left(\beta, x_{0}\right)$ depends continuously and $L_{T}\left(\beta, x_{0}\right)$ depends upper semi-continously on $\left(\beta, x_{0}\right)$, and $L\left(\beta, x_{0}\right) \subset L_{T}\left(\beta, x_{0}\right)$, the function $\left(\beta, x_{0}\right) \rightarrow d\left(L_{T}\left(\beta, x_{0}\right), L\left(\beta, x_{0}\right)\right)$ is u.s.c. on $\left[\beta_{0}, 1\right] \times X$. One also has $\forall\left(\beta, x_{0}\right), \lim _{T \rightarrow+\infty} d\left(L_{T}\left(\beta, x_{0}\right), L\left(\beta, x_{0}\right)\right)=$

0 . Then, $\forall x_{0}, \forall \beta, \exists T_{0}$ such that $d\left(L_{T_{0}}\left(\beta, x_{0}\right), L\left(\beta, x_{0}\right)\right)<\frac{\varepsilon}{9}$. Moreover, since $\left(\beta, x_{0}\right) \rightarrow d\left(L_{T_{0}}\left(\beta, x_{0}\right), L\left(\beta, x_{0}\right)\right)$ is u.s.c., there exists a neighborhood $\mathcal{V}\left(\beta, x_{0}\right)$ of $\left(\beta, x_{0}\right)$ such that $\forall\left(\beta^{\prime}, x\right) \in \mathcal{V}\left(\beta, x_{0}\right), d\left(L_{T_{0}}\left(\beta^{\prime}, x\right), L\left(\beta^{\prime}, x\right)\right)<\frac{\varepsilon}{9}$.

Since $L\left(\beta, x_{0}\right) \subset L_{T+1}\left(\beta, x_{0}\right) \subset L_{T}\left(\beta, x_{0}\right), \forall T$, then one finally has that $\forall T \geq$ $T_{0}, \forall\left(\beta^{\prime}, x\right) \in \mathcal{V}\left(\beta, x_{0}\right), d\left(L_{T}\left(\beta^{\prime}, x\right), L\left(\beta^{\prime}, x\right)\right)<\frac{\varepsilon}{9}$. But, since $\left[\beta_{0}, 1\right] \times X$ is a compact set, $T_{0}$ can be chosen independently of the neighborhood $\mathcal{V}\left(\beta, x_{0}\right)$ and one obtains $\forall \varepsilon>0, \exists T_{0}$ such that $\forall T \geq T_{0}, \forall\left(\beta, x_{0}\right) \in\left[\beta_{0}, 1\right] \times X, d\left(L_{T}\left(\beta, x_{0}\right), L\left(\beta, x_{0}\right)\right)<$ $\frac{\varepsilon}{9}$.

(ii) Let us show that the sets $L_{T}\left(\beta, x_{0}\right)$ are equicontinuous.

We already know that $L\left(\beta, x_{0}\right)$ is continuous and compact-valued. Let $\varepsilon>0$. Then the compact set $\left[\beta_{0}, 1\right] \times X$ may be covered by a finite number of open neighborhood $\left(\mathcal{V}_{i}\right)_{i=1}^{m}$ such that $\forall\left(\beta, x_{0}\right),\left(\beta^{i}, x_{0}^{i}\right) \in \mathcal{V}_{i}, d\left(L\left(\beta, x_{0}\right), L\left(\beta^{i}, x_{0}^{i}\right)\right)<\frac{\varepsilon}{9}$. Then using the triangle inequality with (i), one obtains that $\forall T \geq T_{0}, d\left(L_{T}\left(\beta, x_{0}\right), L_{T}\left(\beta^{i}, x_{0}^{i}\right)\right)<$ $\frac{\varepsilon}{3}$. 
(iii) Uniform approximation of the sets $G_{T}^{\beta}\left(x_{0}\right)$ to $L_{T_{0}}\left(\beta, x_{0}\right)$

Let

$$
G_{T}^{\beta}\left(x_{0}\right)=\left\{\left(a_{0}, a_{1}\right) \in \mathbb{R}^{2 n} \text { with }\left\|a_{0}\right\|=1 \text { s.t. } a_{1}=D g_{T}^{\beta}\left(x_{0}\right) . a_{0}\right\} .
$$

Observe that

$$
\begin{aligned}
G_{T}^{\beta}\left(x_{0}\right)= & \left\{\left(a_{0}, a_{1}\right) \in \mathbb{R}^{2 n} \text { with }\left\|a_{0}\right\|=1 \text { s.t. there exist } a_{1}, a_{2}, \ldots, a_{T-1}, a_{T}\right. \\
& \text { which satisfy the first-order conditions of lemma } 5\} .
\end{aligned}
$$

By lemma $4,\left\{g_{T}^{\beta, t}\right\}_{T}$ converges uniformly to $g^{\beta, t}, L_{T_{0}}$ is an u.s.c. correspondence, then by lemma 5 , the first order conditions of this lemma and lemma 6 , $\forall\left(\beta, x_{0}\right), \forall \varepsilon>0, \exists T_{1} \geq T_{0}$ such that $\forall T \geq T_{1}, G_{T}^{\beta}\left(x_{0}\right)$ is contained in the ball centered in $L_{T_{0}}\left(\beta, x_{0}\right)$ of radius $\frac{\varepsilon}{3}$.

Moreover, since $g^{\beta, t}$ is continuous and $\left\{g_{T}^{\beta, t}\right\}_{T}$ converges uniformly to $g^{\beta, t}$, then the upper semi-continuity of $L_{T_{0}}$ implies that $\forall \varepsilon>0, \forall\left(\beta, x_{0}\right), \exists \mathcal{V}\left(\beta, x_{0}\right)$ a neighborhood of $\left(\beta, x_{0}\right)$ and $T_{1}$ such that $\forall\left(\beta^{\prime}, x\right) \in \mathcal{V}\left(\beta, x_{0}\right), \forall T \geq T_{1}, G_{T}^{\beta^{\prime}}(x)$ is contained in the ball centered in $L_{T_{0}}\left(\beta, x_{0}\right)$ of radius $\frac{\varepsilon}{3}$.

Then, using if necessary a finer finite open cover than $\left(\mathcal{V}_{i}\right)_{i=1, \ldots, m}$, one has that $\forall \mathcal{V}_{i}, \exists\left(\beta^{i}, x_{0}^{i}\right), \exists T_{1} \geq T_{0}$ such that $\forall\left(\beta, x_{0}\right) \in \mathcal{V}_{i}, \forall T \geq T_{1}, G_{T}^{\beta}\left(x_{0}\right)$ is contained in the ball centered in $L_{T_{0}}\left(\beta^{i}, x_{0}^{i}\right)$ of radius $\frac{\varepsilon}{3}$.

Moreover, the compactness of $\left[\beta_{0}, 1\right] \times X$ allows us to choose $T_{1}$ independently of $\mathcal{V}_{i}$.

(iv) $G_{T}^{\beta}\left(x_{0}\right)$ converge uniformly to $L\left(\beta, x_{0}\right)$.

Let $\left(\beta, x_{0}\right)$ be in $\left[\beta_{0}, 1\right] \times X$. Then there exists $i$ such that $\left(\beta, x_{0}\right) \in \mathcal{V}_{i}$, and then one has that $\forall T \geq T_{1}, G_{T}^{\beta}\left(x_{0}\right)$ is contained in the ball centered in $L_{T_{0}}\left(\beta^{i}, x_{0}^{i}\right)$ of radius $\frac{\varepsilon}{3}$. Moreover, by (i) and (ii), one has $d\left(L_{T_{0}}\left(\beta, x_{0}\right), L\left(\beta, x_{0}\right)\right)<\frac{\varepsilon}{9}$ and $d\left(L_{T_{0}}\left(\beta^{i}, x_{0}^{i}\right), L_{T_{0}}\left(\beta, x_{0}\right)\right)<\frac{\varepsilon}{3}$. Then, $\forall T \geq T_{1}, \forall\left(\beta, x_{0}\right), G_{T}^{\beta}\left(x_{0}\right)$ is contained in the ball centered in $L\left(\beta, x_{0}\right)$ of radius $\varepsilon$.

(v) Define the matrix $M$ as the following: its $i$ th column is the unique vector $b_{i} \in \mathbb{R}^{n}$ such that $\left(a_{i}, b_{i}\right) \in L\left(\beta, x_{0}\right)$ with $a_{i}=\left(0,0, \ldots, 0,1_{(i)}, 0, \ldots, 0\right)$. Then, (iv) implies that the sequence of matrix $\left\{D g_{T}^{\beta}\left(x_{0}\right)\right\}_{T>1}$ converges uniformly to the matrix $M$. Then, that is $M=D g^{\beta}\left(x_{0}\right)$, and $(\beta, x) \rightarrow D g^{\beta}(x)$ is continuous in any $\beta \in\left[\beta_{0}, 1\right], x \in X$. 


\section{References}

[1] Araujo A., The once but not twice differentiability of the policy function, Econometrica 59 (1991), 1383-1391.

[2] Araujo A., J.A.Scheinkman, Smoothness, comparative dynamics and the turnpike property, Econometrica 45,(1977), 601-620.

[3] Benveniste L.M., J.A.Scheinkman, On the differentiability of the value function, Econometrica 47,(1979), 727-732.

[4] Blot J., Crettez B., On the smoothness of the optimal paths (1999), Manuscript.

[5] Boldrin M., Montrucchio L., On the Differentiability of the Policy Function, Unpublished Manuscript (1988).

[6] Dana R.A., Le Van C., On the Bellman Equation of the Overtaking Criterion, Journal of Optimization Theory and Applications 67,vol.3, (1990).

[7] Gale D., On Optimal Development in a multi-sector economy, Rev.Econ. Studies 34 (1967).

[8] Le Van C., Dana R.A., Dynamic Programming in Economics , Kluwer Academic Publishers, (2002, forthcoming).

[9] McKenzie L.W, Optimal economic growth, turnpike theorems and comparative dynamics, in Arrow K.J., Intriligator M.D. , Handbook of Mathematical Economics, Vol.III, North-Holland, Amsterdam (1986).

[10] Montrucchio L., Lipschitz continuous policy functions, Journal of Mathematical Economics 16 (1987), 259-273.

[11] Montrucchio L., Dynamic Complexity of Optimal Paths and Discount Factors for Strongly Concave problems, Journal of Optimization Theory and Applications 80 (1994), 385-406.

[12] Montrucchio L., Thompson metric,contraction property and differentiability of policy functions, J. Econ. Behavior and Organization 33 (1998), 449-466.

[13] Santos M.S., Smoothness of the policy function in discrete time economic models, Econometrica 5 (1991), 1365-1382.

[14] Santos M.S., Differentiability and comparative analysis in discrete-time infinite-horizon optimization, Journal of Economic Theory 57 (1992), 222229. 
[15] Santos M.S., Smooth dynamics and computation in models of economic growth, Journal of Economic Dynamics and Control 18 (1994), 879-895.

[16] Santos M.S., J. Vigo-Agular, Analysis of a numericaldynamical programming algorithm applied to economic model, Econometrica 66, $\mathrm{N}^{\circ} 2$ (1998), 409-426.

[17] Scheinkman J.A., On optimal steady state on $n$-sector growth models when utility is discounted Journal of Economic Theory 12 (1976), 11-30.

[18] Venditti, A., Strong concavity properties of indirect utility functions in multisector optimal growth models, Journal of Economic Theory 74 (1997), 349367.

[19] Yano, M., On the dual stability of a Von Neumann Facet and the inefficacy of temporary fiscal policy, Econometrica 66 (1998), 427-451. 